\title{
Evaluation of Manual and Automatic Manually Triggered Ventilation Performance and Ergonomics Using a Simulation Model
}

\author{
Nicolas Marjanovic MD, Soizig Le Floch MD, Morgan Jaffrelot MD, and Erwan L’Her MD PhD
}

BACKGROUND: In the absence of endotracheal intubation, the manual bag-valve-mask (BVM) is the most frequently used ventilation technique during resuscitation. The efficiency of other devices has been poorly studied. The bench-test study described here was designed to evaluate the effectiveness of an automatic, manually triggered system, and to compare it with manual BVM ventilation. METHODS: A respiratory system bench model was assembled using a lung simulator connected to a manikin to simulate a patient with unprotected airways. Fifty health-care providers from different professional groups (emergency physicians, residents, advanced paramedics, nurses, and paramedics; $n=10$ per group) evaluated manual BVM ventilation, and compared it with an automatic manually triggered device (EasyCPR). Three pathological situations were simulated (restrictive, obstructive, normal). Standard ventilation parameters were recorded; the ergonomics of the system were assessed by the health-care professionals using a standard numerical scale once the recordings were completed. RESULTS: The tidal volume fell within the standard range (400$600 \mathrm{~mL}$ ) for $25.6 \%$ of breaths $(0.6-45$ breaths) using manual BVM ventilation, and for $28.6 \%$ of breaths $(0.3-80$ breaths) using the automatic manually triggered device (EasyCPR) $(P<.0002)$. Peak inspiratory airway pressure was lower using the automatic manually triggered device (EasyCPR) $\left(10.6 \pm 5\right.$ vs $\left.15.9 \pm 10 \mathrm{~cm} \mathrm{H}_{2} \mathrm{O}, P<.001\right)$. The ventilation rate fell consistently within the guidelines, in the case of the automatic manually triggered device (EasyCPR) only (10.3 \pm 2 vs $17.6 \pm 6, P<.001)$. Significant pulmonary overdistention was observed when using the manual BVM device during the normal and obstructive sequences. The nurses and paramedics considered the ergonomics of the automatic manually triggered device (EasyCPR) to be better than those of the manual device. CONCLUSIONS: The use of an automatic manually triggered device may improve ventilation efficiency and decrease the risk of pulmonary overdistention, while decreasing the ventilation rate. Key words: manual ventilation; bag-valve-mask; automated system; performance evaluation; ergonomy; simulation. [Respir Care 2014;59(5):735-742. (C) 2014 Daedalus Enterprises]

\section{Introduction}

Ventilation guidelines during resuscitation were specified in the most recent consensus from the European Resuscitation Council ${ }^{1}$ and the American Heart Association. ${ }^{2}$

\footnotetext{
Drs Marjanovic, Le Floch, Jaffrelot, and L'Her are affiliated with the Emergency Department, Pôle Arsibou, Centre Hospitalier Universitaire de la Cavale Blanche, 29609 Brest, France. Drs Jaffrelot and L'Her are affiliated with the Centre de Simulation en Santé, LaTim Institut National de la Santé et de la Recherche Médicale, Unité Mixte de Recherche 1101, Université de Bretagne Occidentale, Université Européenne de Bretagne, 29609 Brest, France.
}

The authors have disclosed a relationship with Weinmann. The authors have disclosed no other conflicts of interest.
The bag-valve-mask (BVM) is an essential device for the provision of ventilation in the absence of endotracheal intubation. However, this ventilation technique may be difficult to manage in emergency situations, even for trained teams. The BVM may lead to a large variation in the insufflated tidal volume $\left(\mathrm{V}_{\mathrm{T}}\right)^{3,4}$ and may require twohanded resuscitation to achieve efficiency. ${ }^{1,2,4,5}$ Whereas insufficient $\mathrm{V}_{\mathrm{T}}$ does not allow correct hematosis to be

\footnotetext{
Correspondence: Erwan L'Her MD PhD, Service d'Accueil des Urgences, Centre Hospitalier Universitaire de la Cavale Blanche, Boulevard Tanguy-Prigent, 29609 Brest Cedex, France.E-mail: erwan.lher@chu-brest.fr.
}

DOI: $10.4187 /$ respcare. 02557 


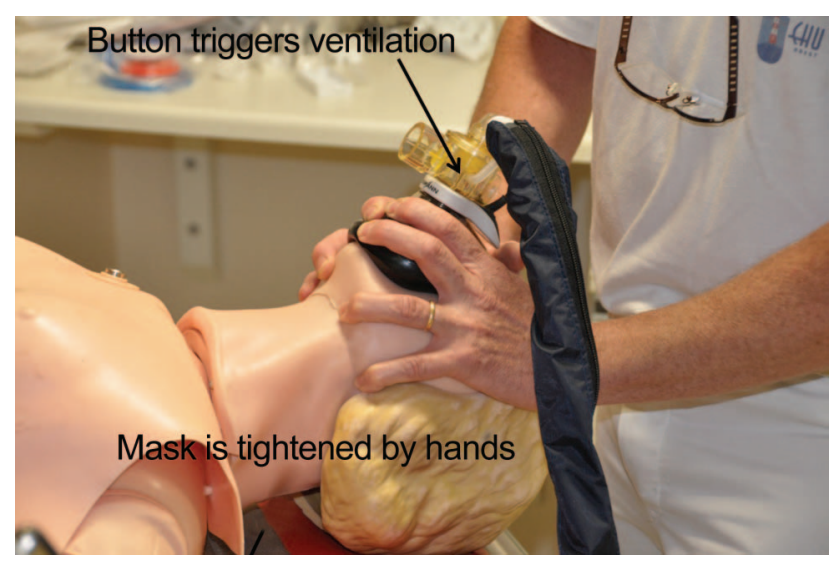

Fig. 1. The automatic manually triggered device (EasyCPR) in use on the simulation model. The mask can be tightened to the face of the model with both hands, thus decreasing leaks and improving mandibular subluxation; ventilation is triggered pressing the knob on the mask with the thumb.

maintained, excessive $\mathrm{V}_{\mathrm{T}}$ may be responsible for pulmonary overdistention and gastric inflation. ${ }^{6}$

The objectives of this bench-test study were to assess the effectiveness of an automatic, but manually triggered, ventilation system, and to evaluate the ability of healthcare professionals to provide guideline-based standard ventilatory parameters.

\section{Methods}

Formal ethical approval was not deemed necessary by the local ethics committee.

\section{Materials}

Fifty providers from five different groups of professional health-care professionals (paramedics, emergency department nurses, senior emergency physicians, emergency medicine residents, and advanced paramedics; $n=10$ per group) were included in the study. Each professional health-care provider evaluated both ventilation systems, a BVM (AMBU Silicone Resuscitator adult, 1.5 L) and an automatic manually triggered device (Medumat EasyCPR, Weinmann Geräte für Medizin, Hamburg, Germany), in a randomized order. The advanced paramedics category included specialized nurses dedicated to prehospital care with training in endotracheal intubation and ventilation.

The automatic manually triggered device (EasyCPR) is a voice-guided device designed to assist first aid responders and health-care providers with emergency ventilation and cardiopulmonary resuscitation (CPR) procedures. The CPR mode features a metronome function, which provides CPR at the correct frequency (not tested in this study) and ventilation assistance that can be manually triggered at the

\section{QUICK LOOK}

\section{Current knowledge}

Manual ventilation with a self-inflating bag and face mask is commonly the first method of ventilation following cardiac arrest. A litany of issues conspire to obfuscate successful manual ventilation including caregiver skill, hand size, fatigue, mask fit, and device design.

\section{What this paper contributes to our knowledge}

The use of an automatic manually triggered device may improve ventilation efficiency and decrease the risk of pulmonary overdistention, while decreasing the ventilation rate.

mask (Fig. 1). Ventilation can be set with a rotary dial, and is time-controlled and volume-constant (fixed respiratory rate for a set volume). When the triggering is activated, $\mathrm{V}_{\mathrm{T}}$ is automatically delivered at the set respiratory rate. Prior to the study, the health-care professionals were given a short demonstration of the technique used to trigger ventilation. The most significant difference between the automatic manually triggered device (EasyCPR) and a standard BVM is that with the former, health-care professionals can keep both hands on the face mask.

A respiratory system analog was assembled using a lung simulator (ASL5000, Ingmar Med) connected to a standard resuscitation manikin (Resusci Anne, Laerdal Medical), thus simulating a patient with unprotected airways. The lung simulator allows mimicking clinically pertinent respiratory mechanics, and the manikin allows the skills of health-care professionals to be evaluated when fitting the mask to the patient's face and performing ventilation. Although chest rise assessment is not possible with the model providers can check ventilation curves on the screen.

The same face mask was used for both situations and devices. Flow and pressure variations were monitored using the lung simulator (ASL5000) sensors. Overall pulmonary distention was assessed by monitoring the position of the lung simulator (ASL5000) piston at the end of expiration. Calibration of the respiratory system analog was performed according to standard procedures.

\section{Procedures}

Different Respiratory Mechanics Patterns. Three different mechanical respiratory patterns were simulated in an apneic patient. Both apparatuses (BVM and the automatic manually triggered ventilation system [EasyCPR]) were tested by each individual during three 1-min sequences 


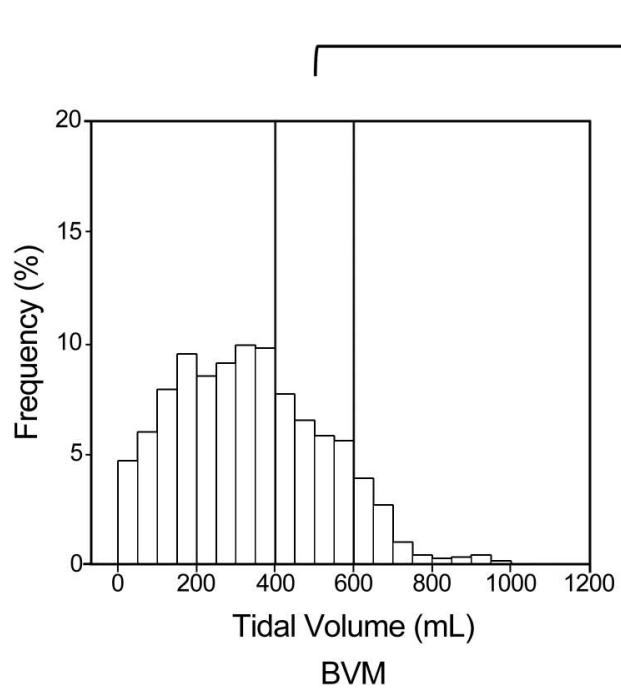

$\mathrm{P}<0.001$

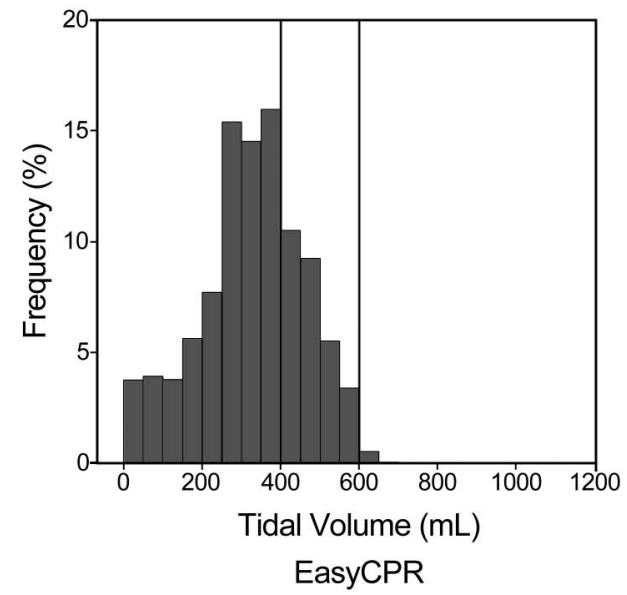

Fig. 2. Tidal volume repartition within all simulation sequences. The figure depicts tidal volume repartition for all groups and recordings. The bold vertical lines represent range values $(400-600 \mathrm{~mL}) . P \leq .05$ was considered significant. $V_{\mathrm{T}}$ was more frequently measured within the range while using an automatic manually triggered device (EasyCPR) compared with BVM (29\% vs 26\% of the measurements, $P<.001)$.

using the following different values of resistance and compliance: (1) high compliance and resistance, designed to simulate a patient with severe COPD ("Obstructive"; resistance $[\mathrm{R}]=20 \mathrm{~cm} \mathrm{H}_{2} \mathrm{O} / \mathrm{L} / \mathrm{s}$, compliance $[\mathrm{C}]=120$ $\mathrm{mL} / \mathrm{cm} \mathrm{H}_{2} \mathrm{O}$ ); (2) normal compliance and resistance ("Normal"; $\mathrm{R}=5 \mathrm{~cm} \mathrm{H} \mathrm{H}_{2} \mathrm{O} / \mathrm{L} / \mathrm{s}, \mathrm{C}=70 \mathrm{~mL} / \mathrm{cm} \mathrm{H}_{2} \mathrm{O}$ ); and (3) normal resistance and low compliance ("Restrictive"; $\mathrm{R}=$ $5 \mathrm{~cm} \mathrm{H} \mathrm{H}_{2} \mathrm{O} / \mathrm{L} / \mathrm{s}, \mathrm{C}=30 \mathrm{~mL} / \mathrm{cm} \mathrm{H}_{2} \mathrm{O}$ ). These sequences were arranged in randomized order. Each sequence was separated from the next by a 1-min rest period.

\section{Assessment of Ergonomics and Respiratory Measure-} ments. The ergonomics of the two devices were scored by each subject and recorded at the end of each complete experimental sequence, using a standardized numerical scale (from 1 [very difficult] to 5 [very easy to use]). Qualitative assessment of the devices was allowed, using short sentences or a small number of words. All quantitative respiratory measurements $\left(\mathrm{V}_{\mathrm{T}}\right.$, end-inspiratory lung volume, peak inspiratory pressure [PIP], and ventilation rate $[\mathrm{VR}]$ ) were performed at atmospheric pressure, constant room temperature $\left(22^{\circ} \mathrm{C}\right)$, and constant lung temperature (cylinder temperature $37^{\circ} \mathrm{C}$ ). Measurements were performed on a mean 5- to 10-cycle period after signal stabilization, using the test lung pressure and flow transducers (ASL 5000, Ingmar, Pittsburgh, Pennsylvania), which we calibrated daily, according to standard procedures. Signal curves were analyzed using the graphics (LabView, National Instruments, Austin, Texas) and the dataacquisition software of the test lung (version SW 3.1). ${ }^{7}$ Flow and pressure transducers are presumed to have a precision of $<10 \mathrm{~mL}$ for volume, and $1 \mathrm{~cm} \mathrm{H}_{2} \mathrm{O}$ for pressure.

\section{Statistical Analysis}

A statistical software package (SPSS for Windows, IBM) was used to perform all analyses. The data are presented as the mean $\pm \mathrm{SD}$, unless specified otherwise. A $P \leq .05$ was considered to indicate a significant result. The nonparametric Mann-Whitney and Wilcoxon tests were performed to compare quantitative values between each group and device. The chi-square test was used to compare $\mathrm{V}_{\mathrm{T}}$ distributions within groups.

\section{Results}

\section{Ventilation Parameters}

The mean inspiratory $\mathrm{V}_{\mathrm{T}}$ was measured below $500 \mathrm{~mL}$ with both devices and for all pathological conditions, and within the range from 400 to $600 \mathrm{~mL}$ for a small number of recordings (Fig. 2). The $\mathrm{V}_{\mathrm{T}}$ distribution was significantly different for both devices and was highly heterogeneous when either different professional health-care groups or different pathological sequences were compared (Table 1). The residual volume was higher for all sequences making use of the BVM $(156 \pm 222$ vs $54 \pm 8 \mathrm{~mL}, P<.001)$.

Figure 3 compares the individual end-inspiratory lung volume distributions for the BVM and the automatic manually triggered device (EasyCPR), during the Obstructive pattern sequences. 
Automatic Versus Manual Ventilation Performance

Table 1. Tidal Volume Cycle Repartition Within the 400-600 mL Range for Each Device, Group, and Simulation Sequence

\begin{tabular}{|c|c|c|c|c|}
\hline \multirow{2}{*}{$\begin{array}{c}\quad \text { Group } \\
(n=10 \text { per group })\end{array}$} & \multirow{2}{*}{ Sequence } & \multicolumn{2}{|c|}{$\mathrm{V}_{\mathrm{T}}{ }^{*}$} & \multirow{2}{*}{$P^{\dagger}$} \\
\hline & & BVM & EasyCPR & \\
\hline \multirow[t]{4}{*}{ EMD } & Restrictive & $256 / 676(37.9 \%)$ & $22 / 349(6.3 \%)$ & $<.001$ \\
\hline & Normal & $163 / 608(26.8 \%)$ & $71 / 316(22.5 \%)$ & .15 \\
\hline & Obstructive & $280 / 620(45.2 \%)$ & $129 / 305(42.3 \%)$ & .41 \\
\hline & All sequences & $699 / 1,904(36.7 \%)$ & $222 / 970(22.9 \%)$ & $<.0001$ \\
\hline \multirow[t]{4}{*}{ EMR } & Restrictive & $3 / 509(0.6 \%)$ & $47 / 330(14.2 \%)$ & $<.001$ \\
\hline & Normal & $10 / 516(1.9 \%)$ & $1 / 316(0.3 \%)$ & $0.06 \ddagger$ \\
\hline & Obstructive & $124 / 576(21.5 \%)$ & $104 / 306(34 \%)$ & $<.001$ \\
\hline & All sequences & $137 / 1,601(8.6 \%)$ & $152 / 952(16 \%)$ & $<.0001$ \\
\hline \multirow[t]{4}{*}{$\mathrm{AP}$} & Restrictive & $156 / 527(29.6 \%)$ & $44 / 336(13.1 \%)$ & $<.001$ \\
\hline & Normal & $166 / 531(31.3 \%)$ & 108/291 (37.1\%) & .09 \\
\hline & Obstructive & $214 / 510(42 \%)$ & $194 / 316(61.4 \%)$ & $<.001$ \\
\hline & All sequences & $536 / 1,568(34.2 \%)$ & $346 / 943(36.7 \%)$ & .21 \\
\hline \multirow[t]{4}{*}{ EDN } & Restrictive & $40 / 492(8.1 \%)$ & $22 / 266(8.3 \%)$ & .95 \\
\hline & Normal & $88 / 439(20 \%)$ & $84 / 260(32.3 \%)$ & $<.001$ \\
\hline & Obstructive & $128 / 472(27.1 \%)$ & $204 / 254(80.3 \%)$ & $<.001$ \\
\hline & All sequences & $256 / 1,403(18.2 \%)$ & $310 / 780(39.7 \%)$ & $<.0001$ \\
\hline \multirow[t]{4}{*}{ PM } & Restrictive & $167 / 496(33.7 \%)$ & $9 / 341(2.6 \%)$ & $<.001$ \\
\hline & Normal & $142 / 471(30.1 \%)$ & $71 / 337(21.1 \%)$ & .004 \\
\hline & Obstructive & $89 / 480(18.5 \%)$ & $221 / 325(68 \%)$ & $<.001$ \\
\hline & All sequences & $398 / 1,447(27.5 \%)$ & $301 / 1,003(30 \%)$ & .19 \\
\hline Overall cycles & All sequences & $2,026 / 7,923(25.6 \%)$ & $1,331 / 4,648(28.6 \%)$ & $<.0002$ \\
\hline $\begin{array}{l}\text { The automatic manually trig } \\
\text { EMR and EDN, but was not } \\
\text { sequences. } P \leq .05 \text { was con } \\
* \text { Values are reported as } n \text { c) } \\
\dagger \text { Chi-square nonparametric } \\
\text { † Fisher exact test. } \\
\text { EMD = senior emergency } \\
\text { EMR = emergency resident } \\
\text { AP = advanced paramedics } \\
\text { EDN = emergency departm } \\
\mathrm{PM}=\text { paramedics } \\
\mathrm{V}_{\mathrm{T}}=\text { tidal volume }\end{array}$ & $\begin{array}{l}\text { PR) provided more c } \\
\text { and AP. The efficienc } \\
\text { artition range/ } N \text { cycles }\end{array}$ & $\begin{array}{l}\text { compared with the bag-valve- } \\
\text { manually triggered device (E) } \\
\text { f } 400-600 \mathrm{~mL} \text { ). }\end{array}$ & $\begin{array}{l}.6 \% ; P<.0002) \text {. This benefit } \\
\text { lower than the BVM for EMD }\end{array}$ & $\begin{array}{l}\text { eased for } \\
\text { estrictive }\end{array}$ \\
\hline
\end{tabular}

Figure 4 illustrates the differences between the data for the two devices within the different professional healthcare groups, during the Normal pattern sequences. A similar difference distribution was observed for the two devices in the case of the Obstructive sequence. The VR was consistent with guidelines while using the automatic manually triggered device (EasyCPR), between 9 and 11 breaths/min for $93 \%$ of the recordings (see Fig. 4), but not with BVM $(10.3 \pm 2$ vs $17.6 \pm 6$ breaths $/ \mathrm{min}$, respectively, $P<.001)$.

Peak inspiratory airway pressure was lower using the automatic manually triggered device (EasyCPR) (10.6 \pm 5 vs $\left.15.9 \pm 10 \mathrm{~cm} \mathrm{H}_{2} \mathrm{O}, P<.001\right)$.

\section{Device Ergonomics}

The ergonomics of the automatic manually triggered device (EasyCPR) were considered to be superior by two professional health-care groups (emergency department nurses and paramedics) when compared to those of the $\operatorname{BVM}(P=.04$ and $P=.006$, respectively). The various expressions used to characterize these devices were "tiredness" (7 of 50 health-care professionals; 14\%) and "better control" (3 of 50 health-care professionals; $6 \%$ ) for the BVM, and "better fitting of the mask thanks to the use of both hands," "fewer leaks" (6 of 50 health-care professionals; 12\%), and "fewer feelings" (6 of 50 health-care professionals; $12 \%$ ) for the automatic manually triggered device (EasyCPR).

\section{Discussion}

This experimental bench-test study describes improved compliance with guidelines ${ }^{1}$ when an automatic manually triggered ventilation device is used (lower VR and PIP, more regular $\mathrm{V}_{\mathrm{T}}$ values, less overdistention), when compared with a BVM. 


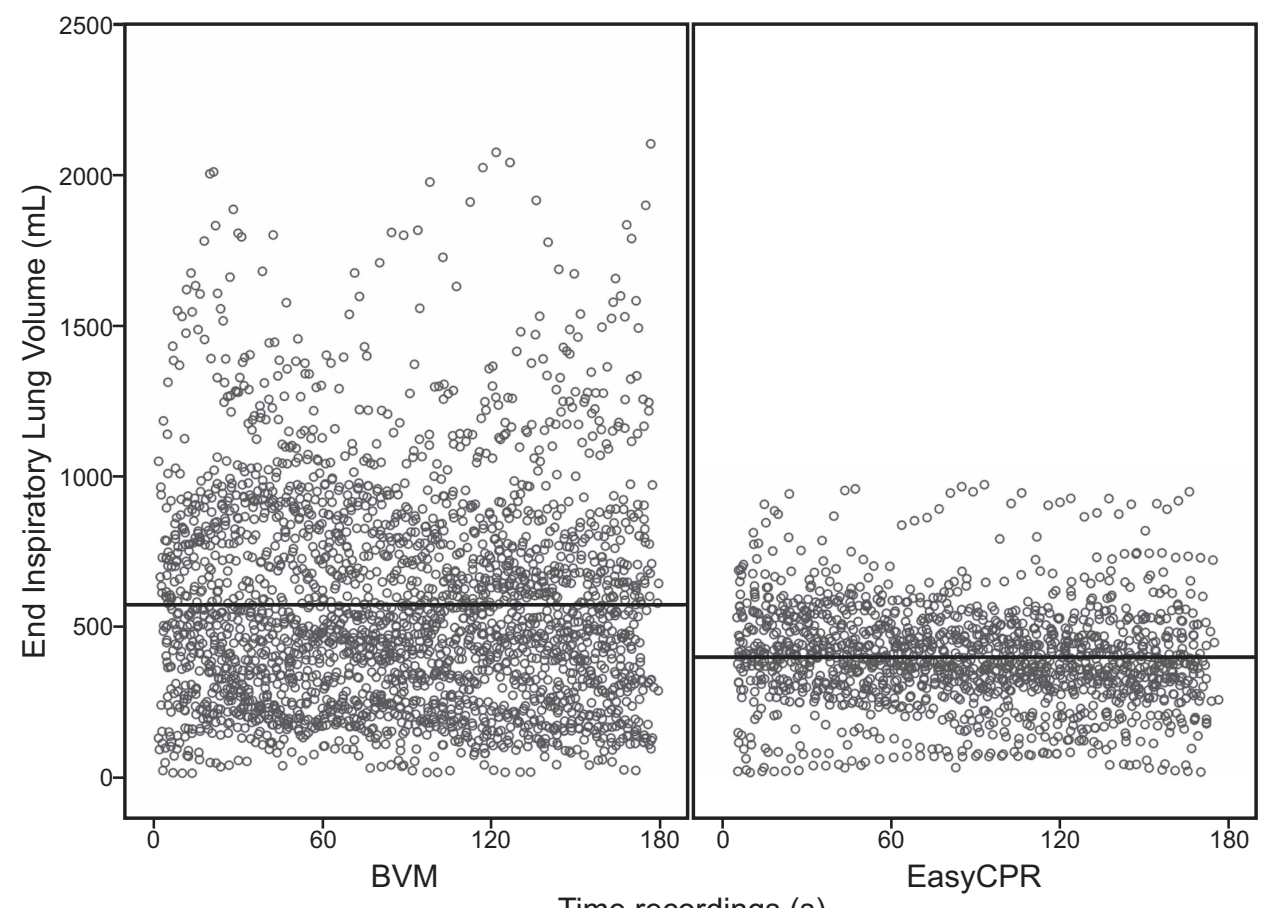

Fig. 3. Individual end-inspiratory lung volume distributions for the BVM and automatic manually triggered device (EasyCPR), during the Obstructive pattern sequences. The figure depicts individual end-inspiratory lung volume distribution during the obstructive sequences, for bag-valve-mask (BVM) and the automatic manually triggered device (EasyCPR). Bold lines corresponded to end-inspiratory lung volume mean values for each device. No significant difference was observed for the mean tidal volume value, but the overall distribution and extreme values favor the use of the automatic manually triggered device (EasyCPR).

Depending on the experimental setting and type of device, contradictory results have been described in the literature. In some cases, a BVM may induce higher peak airway pressure and gastric insufflation, when compared to pressure-cycled, manually triggered devices. ${ }^{8-12}$ In a recent experimental study, an automatic manually triggered device (EasyCPR) did not prove to be superior to $\mathrm{BVM}$ in terms of $\mathrm{V}_{\mathrm{T}}$ values, inspiratory time, and intrapulmonary pressures in a group of 74 medical students. ${ }^{13}$

These differences may arise either from the settings used in the case of pressure-cycled devices, and/or from the use of simulated resistance and compliance settings. In the study by Bergrath et al, ${ }^{13}$ respiratory mechanics of the model were not monitored, and it seems obvious that conditions mimicking COPD were not used, which may have induced more significant differences between the devices. The results found with such trials, combined with the fact that manually triggered devices are much more expensive than BVM devices and require an oxygen source have not allowed the European Resuscitation Council and American Heart Association to provide consistent guidelines concerning the use of manually triggered devices. ${ }^{14}$

To the best of our knowledge, the experimental study described here is the first to evaluate multiple ventilation parameter recordings in the context of clinically relevant pathological situations and within various professional health-care groups.

\section{Impact of Single-Handed versus Two-Handed Resuscitation}

In this study, the mean $\mathrm{V}_{\mathrm{T}}$ always remained $<500 \mathrm{~mL}$, whatever the device or type of sequence. Several studies $^{3-5,15}$ have shown that resuscitation using both hands may be more efficient than resuscitation using one hand, in terms of $\mathrm{V}_{\mathrm{T}}$ delivery (higher mean value and lower variation). Our results show that the automatic manually triggered device (EasyCPR) is more efficient in terms of $\mathrm{V}_{\mathrm{T}}$ delivery, since with this device all health-care professionals used both hands to keep the mask in place and control leaks. However, the mean $\mathrm{V}_{\mathrm{T}}$ always remained at $<500 \mathrm{~mL}$ and fell outside the range limit $(400-600 \mathrm{~mL}$ ) for less than $72 \%$ of the recordings, whatever the device used. With the automatic manually triggered device (EasyCPR), the $\mathrm{V}_{\mathrm{T}}$ can be easily modified by adjusting certain settings (which are, however, related to the VR), whereas BVM ventilation requires the presence of a second rescuer and regular training. 

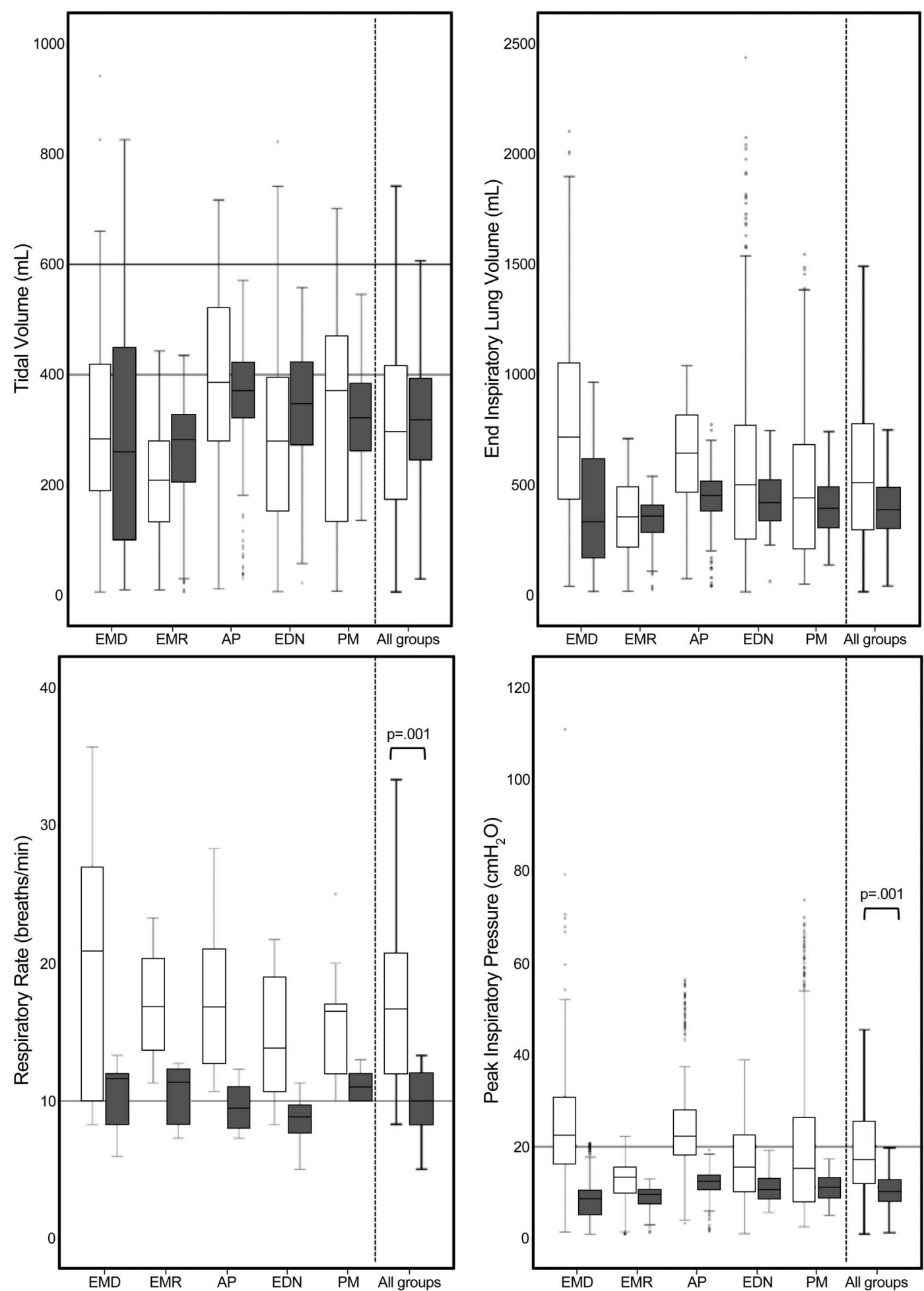

Fig. 4. Box and whisker plot for respiratory measurements during the Normal pattern sequences. Tidal volume, end-inspiratory lung volume, ventilation rate, and peak inspiratory pressure distribution during the Normal pattern sequences recordings. The boundaries on the box indicate the 25th and 75th percentiles, and the line within the box indicates the median. Whiskers above and below the box indicate the 90th and 10th percentiles. Circles represented outlying values. Stars represented exceptional values. Bold lines correspond to range values for tidal volume, lower esophageal sphincter opening pressure, peak inspiratory pressure, and recommended values for residual volume. $\mathrm{EMD}=$ senior emergency physicians; EMR= emergency residents-juniors; AP= advanced paramedics; EDN= emergency department nurses; PM= paramedics; $n=10$ for each group; $P \leq .05$ was considered significant. A significant difference was observed between the devices in terms of VR and PIP, the two being lower and more consistent with guidelines while using the automatic manually triggered device (EasyCPR). 
Although there is no statistical difference between the two types of devices in terms of mean $\mathrm{V}_{\mathrm{T}}$, a very broad $\mathrm{V}_{\mathrm{T}}$ distribution was observed for the BVM.

\section{VR}

Several clinical studies ${ }^{16,17}$ have demonstrated that hyperventilation and/or ventilation at a rate higher than the standard recommendation was frequent in patients with cardiac arrest, both outside and within the hospital. Our data are consistent with such findings, no matter what professional health-care group is reporting the data. Conversely, the VR values obtained with the automatic manually triggered device (EasyCPR) were more consistent, as a result of its controlled VR. This outcome may be of importance, since hyperinflation is a known problem in CPR because it increases intrathoracic pressure, which reduces the hemodynamic effectiveness of chest compressions. ${ }^{16}$ In experimental animal studies, Aufderheide and Lurie $^{18}$ and Aufderheide et al ${ }^{19}$ demonstrated that hyperventilation decreased coronary perfusion pressures and arterial blood pressure, thus resulting in lower survival rates.

\section{Total Volume, Residual Volume, and PIP}

To the best of our knowledge, this is the first study to have concomitantly examined $\mathrm{V}_{\mathrm{T}}$ delivery and overall pulmonary distention during resuscitation. Extremely variable delivery volumes were observed with the manual device, whereas more regular volumes were delivered while using the automatic device. This difference can be at least partially explained by the differences between the two devices, in terms of VR and therefore expiratory time variation. As a consequence, residual volume and overall distention may also increase, thus making ventilation more difficult due to higher intrapulmonary pressures and increased leakage.

Gastric inflation and opening of the lower esophagus sphincter is clearly related to PIP. ${ }^{6,20}$ Very few experimental studies have evaluated gastric inflation during CPR. Osterwalder and Schuhwerk ${ }^{9}$ demonstrated differences in terms of gastric inflation proportion between the BVM and automatic ventilators ( $42 \%$ vs $0 \%$ ) in a manikin study, and explained this difference by a higher PIP while using a BVM. In another study, ${ }^{21}$ a decrease in lower esophagus sphincter opening pressure within the first minutes of cardiac arrest was suggested. Although such gastric inflation was not recorded during our study, higher gastric inflation could have occurred during use of a BVM or automatic manually triggered device (EasyCPR) device due to a significantly increased PIP.

\section{Differences Among the Three Different Pathological Situations}

Few experiments have detailed the differences that could be induced during manual ventilation, according to patients' respiratory mechanics. The results presented herein provide new insights about the major differences that are to be expected among the patients even if all of these differential effects are consistent with standard knowledge on respiratory mechanics.

Besides the strict application of guidelines our results are consistent with the fact that in real patients with pulmonary diseases, VR should be adjusted. In a patient with COPD, a VR equal to or below the guideline level (10 breaths/min) should be a major goal, while an increase of the rate immediately induces hyperinflation and elevated peak pressure; in a Restricted patient, an increased VR may not have such deleterious effects and may in fact increase minute ventilation, while $\mathrm{V}_{\mathrm{T}}$ values are usually smaller.

\section{Study Limitations}

Several limitations of this study should be emphasized. First, although care was taken to ensure correct respiratory mechanics, the experimental settings may have been different from those encountered in real-life situations. In physiological terms, the patient-to-mask interface and airways are not strictly identical to those observed in real patients. Even though we carefully chose the components used in our respiratory analog, these may not have exactly duplicated in vivo ventilation and the variability encountered with real patients. Second, not all of the parameters of interest could be integrated into our evaluation due to the specific design of the respiratory analog. The absence of chest rise due to direct connection of the manikin airways to the lung simulator may have modified the healthcare professional ventilation procedures, whereas most training courses use chest rise as the sole indicator of ventilation adequacy. Nevertheless, this specificity of the model was emphasized at the beginning of the experiment, and the health-care professionals were able to check the ventilation curves. Third, we may also consider that the small number of providers in each group makes comparison difficult. For this reason, most of our analysis was focused on the overall results (50 subjects), rather than on individual differences.

\section{Conclusions}

The use of an automatic, manually triggered ventilation device for resuscitation may present valuable advantages over the standard manual BVM ventilation. Such devices may improve ventilation efficiency and decrease the risk 
of pulmonary overdistention while at the same time decreasing VR. Clinicians should, however, be aware that the performance of such devices depends strongly on each patient's pathology and on the user's individual experience. It is essential that in vivo studies be implemented to evaluate the potential impact of automatic manually triggered devices in the clinical setting.

\section{REFERENCES}

1. Deakin CD, Nolan JP, Soar J, Sunde K, Koster RW, Smith GB, et al. European Resuscitation Council Guidelines for Resuscitation 2010 Section 4. Adult advanced life support. Resuscitation 2010;81(10): 1305-1352.

2. Neumar RW, Otto CW, Link MS, Kronick SL, Shuster M, Callaway CW, et al. Part 8: adult advanced cardiovascular life support: 2010 American Heart Association Guidelines for Cardiopulmonary Resuscitation and Emergency Cardiovascular Care. Circulation 2010; 122(18 Suppl 10):S729-S767.

3. Seidelin PH, Stolarek IH, Littlewood DG. Comparison of six methods of emergency ventilation. Lancet 1986;2(8518):1274-1275.

4. Lee HM, Cho KH, Choi YH, Yoon SY. Can you deliver accurate tidal volume by manual resuscitator? Emerg Med J 2008;25(10):632634.

5. Jesudian MC, Harrison RR, Keenan RL, Maull KI. Bag-valve-mask ventilation; two rescuers are better than one: preliminary report. Crit Care Med 1985;13(2):122-123.

6. Wenzel V, Idris AH, Banner MJ, Kubilis PS, Williams JL Jr. Influence of tidal volume on the distribution of gas between the lungs and stomach in the nonintubated patient receiving positive-pressure ventilation. Crit Care Med 1998;26(2):364-368.

7. L'Her E, Roy A. Bench tests of simple, handy ventilators for pandemics: performance, autonomy, and ergonomy. Respir Care 2011; 56(6):751-760.

8. von Goedecke A, Wenzel V, Hörmann C, Voelckel WG, WagnerBerger HG, Zecha-Stallinger A, et al. Effects of face mask ventilation in apneic patients with a resuscitation ventilator in comparison with a bag-valve-mask. J Emerg Med 2006;30(1):63-67.

9. Osterwalder JJ, Schuhwerk W. Effectiveness of mask ventilation in a training mannikin. A comparison between the Oxylator EM100 and the bag-valve device. Resuscitation 1998;36(1):23-27.
10. Stallinger A, Wenzel V, Wagner-Berger H, Schäfer A, Voelckel WG, Augenstein S, et al. Effects of decreasing inspiratory flow rate during simulated basic life support ventilation of a cardiac arrest patient on lung and stomach tidal volumes. Resuscitation 2002;54(2):167-173.

11. Menegazzi JJ, Winslow HJ. In-vitro comparison of bag-valve-mask and the manually triggered oxygen-powered breathing device. Acad Emerg Med 1994;1(1):29-33.

12. Updike G, Mosesso VN Jr., Auble TE, Delgado E. Comparison of bag-valve-mask, manually triggered ventilator, and automated ventilator devices used while ventilating a nonintubated mannikin model. Prehosp Emerg Care 1998;2(1):52-55.

13. Bergrath S, Rossaint R, Biermann H, Skorning M, Beckers SK, Rörtgen D, et al. Comparison of manually triggered ventilation and bag-valve-mask ventilation during cardiopulmonary resuscitation in a manikin model. Resuscitation 2012;83(4):488-493.

14. Cave DM, Gazmuri RJ, Otto CW, Nadkarni VM, Cheng A, Brooks SC, et al. Part 7: CPR techniques and devices: 2010 American Heart Association Guidelines for Cardiopulmonary Resuscitation and Emergency Cardiovascular Care. Circulation 2010;122(18 Suppl 3):S720S728.

15. Wheatley S, Thomas AN, Taylor RJ, Brown T. A comparison of three methods of bag valve mask ventilation. Resuscitation 1997; 33(3):207-210.

16. O'Neill JF, Deakin CD. Do we hyperventilate cardiac arrest patients? Resuscitation 2007;73(1):82-85.

17. Abella BS, Alvarado JP, Myklebust H, Edelson DP, Barry A, O'Hearn $\mathrm{N}$, et al. Quality of cardiopulmonary resuscitation during in-hospital cardiac arrest. JAMA 2005;293(3):305-310.

18. Aufderheide TP, Lurie KG. Death by hyperventilation: a common and life-threatening problem during cardiopulmonary resuscitation. Crit Care Med 2004;32(9 Suppl):S345-S351.

19. Aufderheide TP, Sigurdsson G, Pirrallo RG, Yannopoulos D, McKnite $\mathrm{S}$, von Briesen $\mathrm{C}$, et al. Hyperventilation-induced hypotension during cardiopulmonary resuscitation. Circulation 2004;109(16): 1960-1965.

20. Wenzel V, Idris AH, Banner MJ, Kubilis PS, Band R, Williams JL Jr., Lindner KH. Respiratory system compliance decreases after cardiopulmonary resuscitation and stomach inflation: impact of large and small tidal volumes on calculated peak airway pressure. Resuscitation 1998;38(2):113-118.

21. Bowman FP, Menegazzi JJ, Check BD, Duckett TM. Lower esophageal sphincter pressure during prolonged cardiac arrest and resuscitation. Ann Emerg Med 1995;26(2):216-219. 